\title{
Vergleichende Histologie des Sphincter ani externus.
}

\author{
Von \\ Yosiharu Inaba. \\ (Aus dem Anatomischen Institut der Medizinischen Fakultät zù Okayama ; \\ Vorstand: Prof. M. Seki.)
}

Mit 6 Abbildungen auf Tafel IV.

In der früheren Arbeit ( 1942 b) habe ich beim Menschen Altersveränderungen des Sphincter ani externus beobachtet und bin auf eine Besprechung über die Zusammenhänge zwischen der Funktion und dem Bau desselben eingegangen. Es ist ohne weiteres begreiflich, dass bei Tieren je nach der Verschiedenheit von Habitus und Lebensbedingung die Tätigkeit des Sphincter ani externus variieren kann, was naturgemäss auch $\mathrm{zu}$ voneinander abweichendem Muskelbau führen muss. Wir finden trotzdem im Schrifttum fast gar keine diesbezüglichen Berichte.

\section{Material und Methode.}

Als Untersuchungsmaterial dienten ausser einem Menschen (23 Jahre) to Therarten: Hund (Canis familiaris) ( $5,0 \mathrm{~kg})$, Leopard (Felis pardus) $(33,0$ $\mathrm{kg}$ ), Dachs (Nyctereutes viverrinus) ( $5,0 \mathrm{~kg})$, Kaninchen $(2,0 \mathrm{~kg})$, Fuchs $(\overline{V u l}-$ pes japonicus) (3,7 kg), Reh aus Formosa (Muntiacus reevesii) (9,5 kg), Wolf aus Korea (Canis lupus coreanus) $(18,7 \mathrm{~kg})$, Katze (Felis domestica) $(2,3 \mathrm{~kg})$, Affe (Macacus japonicus) $(4,2 \mathrm{~kg})$ und Seebär (Callotaria ursina) $(\mathbf{2 4}, 0 \mathrm{~kg})$. Von diesen Tieren und dem Menschen wurde der Anus mit dem umgebenden Gewebe exstirpiert, der dann in 10\%iger Formalinlösung fixiert und nach der Methylalkohol-Celloidin-Methode von Seki (i937) in Celloidin eingebettet wurde. Die quer geschnittenen Schnitte des Sphincter ani externus wurden nach der Azanfärbung von Heidenhain und der Resorzin-FuchsinFärbung von Weigert gefärbt. Die Messung des Flächeninhaltes der Quèrschnitte der Muskelfasern wurde mit Hilfe des Zeichenapparates und Planimeters ausgeführt. 


\section{Untersuchungsergebnisse.}

I. Hund $(5,0 \mathrm{~kg})$. In der Dicke der Muskelfasern übertrifft der Hund alle anderen Untersuchungstiere. Die Muskelfasern sind an Querschnitten meistens polygonal (Abb. I) und bilden vom Bindegewebe zusammengeschlossene Muskelbündel. Das Bindegewebe $z$ wischen den Muskelfasern ist nur sehr schwach entwickelt. Die elastischen Fasern lassen sich spärlich nachweisen.

2. Leopard $(33,0 \mathrm{~kg})$. Die Dicke der Muskelfasern beträgt $670 \mu^{2}$. Nächst dem Hund ist also der Leopard von den. dicksten Analmuskelfasern. An Querschnitten bieten die Muskelfasern meistens drei- oder viereckige Form dar. Das interstitielle Bindegewebe ist spärlich. Die Muskelfasern bilden deutliche Bündel.

3. Dachs $(5,0 \mathrm{~kg})$. Der Dicke der Muskelfasern nach kommt dieses Tier gleich nach dem Leopard. Die Muskelfasern sind zumeist viereckig oder rundlich. Man sieht hier auch nur spärliches interstitielles Bindegewebe. Die Muskelbündel sind von der Umgebung scharf abgegrenzt.

4. Kaninchen (2,0 kg). Die Dicke der Muskelfasern $\left(580 \mu^{2}\right)$ ist etwas kleiner als beim Dachs. Die Faserquerschnitte sind meistens drei- oder viereckig. Das Bindegewebe zwischen den Fasern ist spärlich. Die Bündelgrenzen sind nicht sehr scharf.

5. Fuchs $(3,7 \mathrm{~kg})$. Die Dicke der Muskelfasern beträgt $360 \mu^{2}$. Die Muskelfasern sind in Querschnitten meistens oval oder viereckig und bilden Muskelbündel mit scharfer Begrenzung. Das interstitielle Bindegewebe ist mit den elastischen Fasern etwas reichlicher versehen.

6. Reh aus Formosa $(9,5 \mathrm{~kg})$. Die Muskelfasern sind von gleicher Dicke wie beim Fuchs und Wolf. Die Mehrzahl der Muskelfasern ist in Querschnitten rundlich und zu scharf abgesetzten Bündeln vereinigt. Das Bindegewebe zwischen den Muskelfasern ist ziemlich reichlich vorhanden, in ihm sind aber die elastischen Fasern gering.

7. Wolf aus Korea ( $18,7 \mathrm{~kg}$ ). Die Dicke der Muskelfasern beträgt 350 $\mu^{2}$. An Querschnitten sind die Muskelfasern rundlich (Abb. 3) und zu Muskelbündeln zusammengeschlossen. Im mässig entwickelten interstitiellen Bindegewebe sind die elastischen Fasern ziemlich zahlreich zu finden.

8. Katze $(2,3 \mathrm{~kg})$. Die Muskelfasern sind durchschnittlich nur $335 \mu^{2}$ dick. Die meisten Muskelfasern zeigen rundliche Querschnitte und bilden deutlich abgrenzbare Muskelbündel. Das interstitielle Bindegewebe und die elastischen Fasern verhalten sich wie beim Wolf.

9. Affe $(4,2 \mathrm{~kg})$. Die Muskelfasern sind beträchtlich dünn. An Querschnitten sehen sie in der Regel rundlich aus (Abb. 2) und bilden deut- 
, Vergleichende Histologie des Sphincter ani externus.

\begin{tabular}{|c|c|c|c|c|c|c|}
\hline Material & 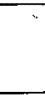 & $\mathrm{M} \pm \mathrm{m}$ & $\sigma$ & V & $\begin{array}{c}\text { Bdg. zw. } \\
\text { d. M.- } \\
\text { fasern }\end{array}$ & $\begin{array}{c}\text { Elast. } \\
\text { Fasern }\end{array}$ \\
\hline Hund $(5,0 \mathrm{~kg})$ & 今 & $7,7^{6} \pm 0,2 x$ & 2,10 & 27,1 & + & + \\
\hline Leopard $(33,0 \mathrm{~kg})$ & 우 & $6,77 \pm 0.35$ & 3,47 & 51,2 & + & + \\
\hline Dachs $(5,0 \mathrm{~kg})$ & 우 & $6,70 \pm 0,25$ & 2,49 & $37, \mathrm{I}$ & + & + \\
\hline Kaninchen $(2,0 \mathrm{~kg})$ & 우 & $5,82 \pm 0,15$ & $I, 51$ & 26,1 & + & + \\
\hline Fuchs $(3,7 \mathrm{~kg})$ & $\widehat{\delta}$ & $3,62 \pm 0,13$ & $I, 26$ & 35,7 & $H$ & $H$ \\
\hline $\operatorname{Reh}(9,5 \mathrm{~kg})$ & $\hat{\delta}$ & $3,58 \pm 0,16$ & 1,63 & 45,5 & $H(\#)$ & $H$ \\
\hline Wolf $(18,7 \mathrm{~kg})$ & 今 & $3,52 \pm 0,17$ & $x, 7 x$ & $4^{8,5}$ & $H$ & $H(\#)$ \\
\hline Katze $(2,3 \mathrm{~kg})$ & 우 & $3,35 \pm 0,12$ & 1,17 & 29,4 & $H$ & + \\
\hline Affe $(4,2 \mathrm{~kg})$ & ऽ & $3,28 \pm 0,13$ & 1,29 & 39,3 & $H$ & \# \\
\hline Seebär $(24,0 \mathrm{~kg})$ & જ & $3,2 I \pm 0, I I$ & $x, 13$ & 35,3 & $H$ & \# \\
\hline Mensch $(50,0 \mathrm{~kg})$ & $\boldsymbol{\delta}$ & $2,23 \pm 0,10$ & $x, 01$ & 42,2 & 册 & H \\
\hline
\end{tabular}

liche Muskelbündel. Zwischen den Muskelfasern ist das Bindegewebe mit zahlreichen elastischen Fasern in mässiger Menge zu sehen.

ro. Seebär $(24,0 \mathrm{~kg})$. Man sieht in Querschnitten rundliche Muskelfasern, die nur $320 \mu^{2}$ dick und $\mathrm{zu}$ scharf abgesetzten Bündeln vereinigt sind. Zwischen den Múskelfasern ist stark elastisch durchsetztes Bindegewebe zu finden (Abb. 4 und 6).

II. Mensch im Alter von 23 Jahren $(50.0 \mathrm{~kg})$. Die Muskelfasern sind $220 \mu^{2}$ dick und am dünnsten unter allen von mir untersuchten Tięren. An Querschnitten sind die Muskelfasern rundlich (Abb. s) und zu scharf abgesetzten Muskelbündeln vereinigt. Zwischen den Muskelfasern findet sich verhältnismässig reichliches Bindegewebe, in welchem auch reichliche elastische Fasern eingeschlossen sind.

\section{Zusammenfassung.}

x. Die Dicke der Muskelfasern des Sphincter ani externus ist bei verschiedenen Tieren sehr verschieden und ordnet sich in der Reihe: Hund> Leopard $>$ Dachs $>$ Kaninchen $>$ Fuchs $\geq$ Reh aus Formosa $\geqq$ Wolf aus Korea $\geqq$ Katze $\geqq A f f e ~ \geqq S e e b a ̈ r ~>M e n s c h$. Bei den Tieren, wie Katze, Affe, Seebär und Mensch, bei welchen die Stuhlentleerung mit längerem Intervall stattfindet oder stattzufinden scheint, besteht also die Muskulatur aus dünneren Fasezn, während die Tiere, wie Hund, Leopard u.a., welche ihren Stuhl häufiger leeren und den Anus nicht lange fest zu verschliessen brauchen, dickere Analmuskelfasern zeigen.

2. Die Menge des Bindegewebes $z$ wischen den Muskelfasern wechselt auch bei den Tieren bedeutend. Im allgemeinen ist es bei dẹm Sphincter mit mehr anhaltender Tätigkeit besser entwickelt und schliesst mehr elastische Fasern ein. 


\section{Literaturverzeichnis.}

In a ba, Y.: Vergleichende Histologie der Atemmuskeln. Okajimas Fol. anat. jap. 2x (1942 a). In aba, Y.: Úber die Altersveränderungen des Sphincter ani externus des Menschen. Okajimas Fol. anat. jap. 21 (1942 b).

Koháshi, Y.: Histologische Untersuchungen der verschiedenen Skeletmuskeln beim Menschen. Okajimas Fol. anat. jap. is (1937).

Seki, M.: Untersuchungen mit nichtwässerigen Flüssigkeiten. III. Erfahrungen auf dem Gebiete der Celloidineinbettung. Z. Zellforsch. 26 (1937).

\section{Erklärung zu Tafel IV.}

Aus den Querschnitten des Sphincter ani externus verschiedener Tiere.

Abb. I Hund. Azanfärbung. Vergt. Isox.

Abb. 2 Affe. Azanfärbung. Vergr. 150X.

Abb. 3 Wolf aus Korea. Azanfärbung. Vergr. IsoX.

Abb. 4 Seebär. Azanfärbung. Vergr. 1 soX.

Abb. 5 Mensch (von 23 Jahren). Azanfärbung. Vergr. 150X.

Abb. 6 Seebār. Resorzinfuchsin-Färbung. Vergr. $300 \times$. 


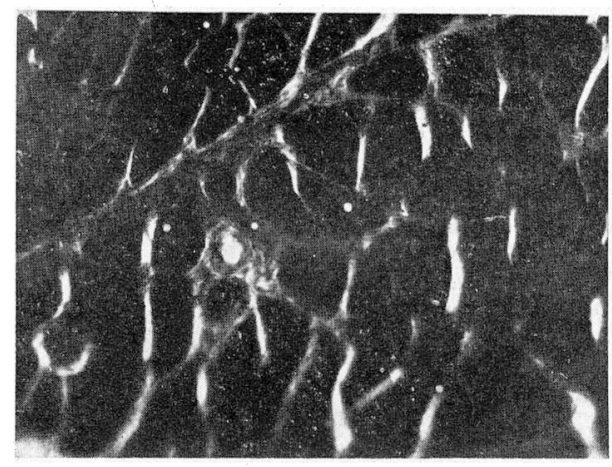

Abb. I.

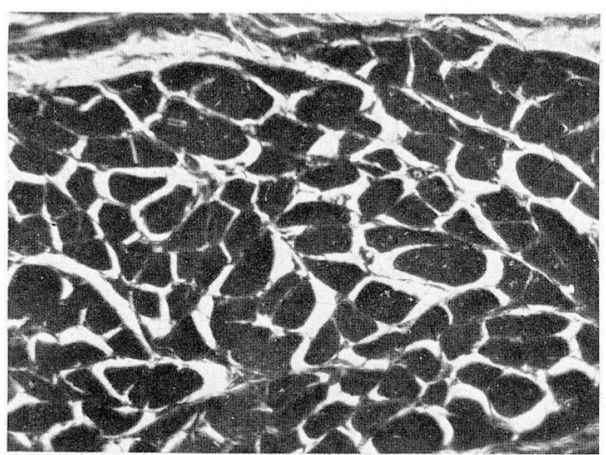

Abb. 3.

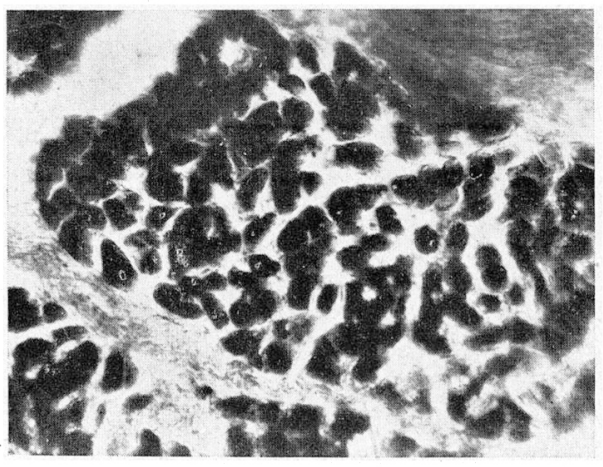

Abb. 5.

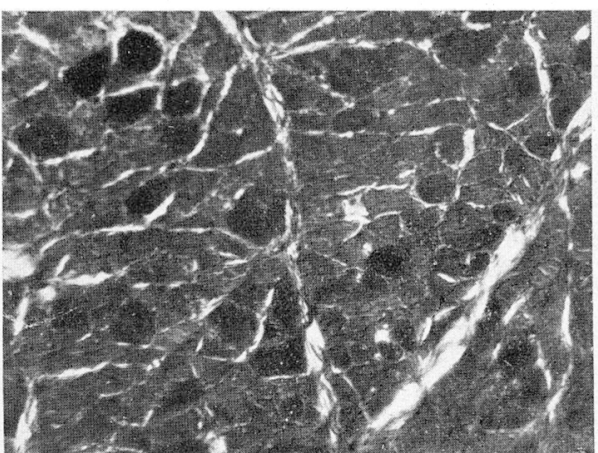

Abb. 2 .

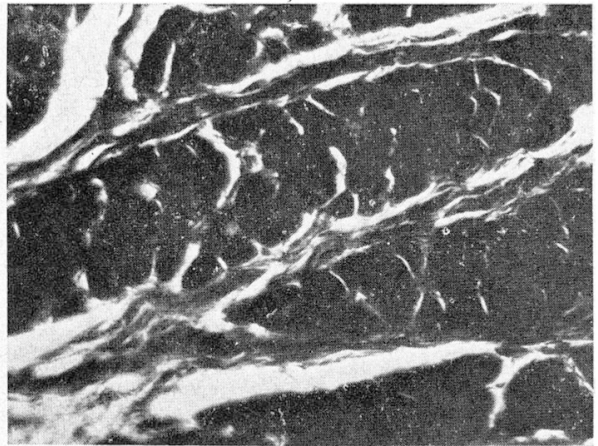

Abb. 4 .

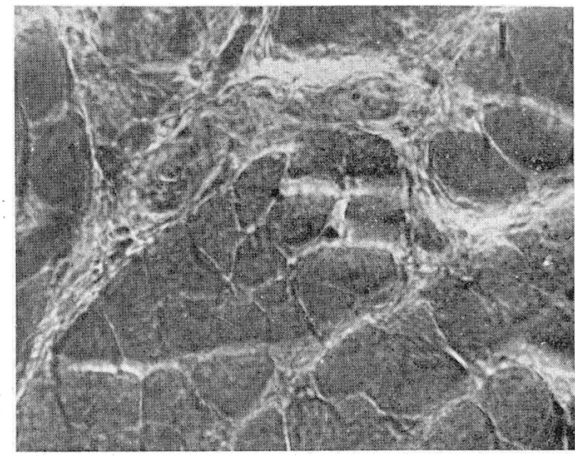

Abb. 6. 